\title{
Optimal Ultrasonic Sensor Configuration Based on Value of Information
}

\author{
Sergio Cantero-Chinchilla \\ Institute for Aerospace Technology \& The Composites Group, The University of Nottingham, UK. \\ E-mail: Sergio.CanteroChinchillal@nottingham.ac.uk
}

Juan Chiachío

Department of Naval Architecture, Ocean \& Marine Engineering, University of Strathclyde, UK. E-mail: juan.chiachio-ruano@strath.ac.uk

Manuel Chiachío

Dept. Structural Mechanics \& Hydraulics Engineering, University of Granada, Spain.

E-mail:mchiachio@ugr.es

Optimal sensor configuration has been proven to be essential in the design of structural health monitoring systems. Its impact in maintenance-related actions in an economic and safety-related way has motivated many authors to investigate this complex problem. In particular, the structural health monitoring systems should be able to provide enough informativeness with a relatively small cost, being affordable for industries. In this paper, such optimality is addressed from the viewpoint of the value of information, combining both the cost and information gain of a structural health monitoring system. In addition, a multiobjective optimization is proposed to address the optimality of the cost-related functions, and its influence in the final decision-making process. The results of the case study show the importance of the definition of a balanced cost-related function. In general, the cubic interpolation spline function defined by two intermediate interpolating points results to be the most appropriate inverse cost functions.

Keywords: Value of information, Bayesian inverse problem, ultrasound, guided-waves, optimal sensor configuration, SHM

\section{Introduction}

Optimal sensor configurations of structural health monitoring (SHM) systems are key for reliable condition-based maintenance. Such optimality should satisfy the required balance between efficiency and informativeness of the sensor configuration. An extremely simple configuration, which only takes into account cost-related factors in the design, would lead to systems that provide a low level of information. However, configurations that pursue the maximization of information with no cost-related restrictions would lead to impractical designs with large sets of sensors. To address the trade-off between cost and information, many authors have proposed the use of the value of information for the design and optimization of SHM systems and operational decisions Konakli et al. (2015); Malings and Pozzi (2016); Straub et al. (2017); Thöns (2018); Malings and Pozzi (2019). Note that the use of the value of information is particularly interesting in the context of ultrasonic guided-waves, where the accuracy of the damage information is highly sensitive to the number of sensors employed.

The issue of optimal sensor configuration for ultrasonic guided-wave based SHM has been par- tially addressed in the literature. Some authors have proposed methodologies to obtain the optimal sensor placement based on geometrical and observational characteristics of both the sensors and the structure, also known as area of coverage Tarhini et al. (2018); Thiene et al. (2016); Khodaei and Aliabadi (2016); Salmanpour et al. (2017). Others have proposed optimization procedures relying on a particular objective function (e.g. probability of detection), which have been addressed by using different techniques such as genetic algorithms (GA), simulated annealing, particle swarm optimization, or artificial neural networks Worden and Burrows (2001); Mallardo et al. (2013); De Stefano et al. (2015); Blanloeuil et al. (2016). However, most of these approaches rely on deterministic approaches, thus limiting its rigorousness and applicability in presence of uncertainties coming from different sources, such as noise, material properties, and epistemic uncertainty, like that coming from the model itself.

The use of Bayesian-related approaches for optimal sensor configuration can be mainly found in structural applications other than ultrasonic guided-waves. In general, probabilistic metrics such as Kullback-Leibler divergence, the

Proceedings of the 29th European Safety and Reliability Conference.

Edited by Michael Beer and Enrico Zio

Copyright (C) 2019 by ESREL2019 Organizers. Published by Research Publishing, Singapore

ISBN: 981-973-0000-00-0 :: doi: 10.3850/981-973-0000-00-0_esrel2019-paper'v01 
Shannon-entropy, or the mutual information have been successfully used for optimizing sensor locations Papadimitriou et al. (2000); Papadimitriou (2004); Argyris et al. (2018); Capellari et al. (2017); Huan and Marzouk (2013). The main conclusion drawn from these works is that the more sensors are added to the system, the more informative the sensor configuration is, hence showing that an additional criterion is needed to obtain a unique optimal sensor configuration, i.e., including position and number of sensors.

In this paper, a hierarchical approach based on value of information is proposed for optimal sensor configuration, which allows optimizing both the number and position of sensors. First, the optimal sensor placement is addressed using the conditional value of information and a greedy algorithm. As a result, the expected information gain is obtained for each optimal sensor layout up to an absolute maximum number of sensors. Then, the optimal sensor configuration can be obtained by using an inverse cost function. The optimality of such cost-related function is further investigated by solving a multiobjective optimization problem. The function that provides the most informative, economic, and identifiable optimal sensor configuration is selected by a nondominated sorting genetic algorithm II (NSGA-II) Deb et al. (2002). A stiffened aluminum plate-like structure with a bounded damage area is used to illustrate the methodology. It is found that costrelated functions based on cubic splines built with two interpolating points are more likely to provide the most balanced optimal sensor configurations.

The rest of the paper is outlined as follows: Section 2 shows the details of the methodology. Section 3 illustrates the methodology with a case study. The results are then discussed in Section 4. Finally, Section 5 provides concluding remarks.

\section{Methodology}

The approach based on value of information for optimal sensor configuration and further search for optimal inverse cost functions is presented throughout this section.

\subsection{Optimal sensor configuration: value of information}

The concept of value of information Howard (1966); Schlaifer and Raiffa (1961) is used here to assess the optimal sensor configuration with regards to the number of sensors. Thus, the required trade-off between information and cost, stemming from manufacturing, installation, and maintenance processes of the SHM system, is rigorously addressed. In general terms, the value of information can be understood as a measure of the benefit produced by the adoption of the optimal sensor configuration compared to the prior state of information, i.e., without the optimal configuration.

In order for the benefit of measuring data to be quantified, a benefit function $b(n, \boldsymbol{\theta})$ that depends on the actual sensor configuration $n$ and the model parameters $\boldsymbol{\theta}$ needs to be defined. Note that the sensor configuration $n$ entails the definition of the optimal sensor layout of the $n$ sensors. Thus, such benefit function is proposed to be proportional to the inverse of cost $f(n)$ of each sensor configuration $n$ and another function $g(\boldsymbol{\theta})$, which accounts for the information gained by the system, such that $b(n, \boldsymbol{\theta}) \propto f(n) g(\boldsymbol{\theta})$.

Next, the concept of maximum prior expected benefit $B^{\prime}$, which is based on the prior information of the model parameters $p(\boldsymbol{\theta})$ Rus et al. (2016), is defined. This is used to obtain the optimal sensor configuration $n_{o p t}^{\prime}$, as follows Konakli et al. (2015):

$$
\begin{aligned}
B^{\prime} & =\mathbb{E}_{p(\boldsymbol{\theta})}\left[b\left(n_{\text {opt }}^{\prime}, \boldsymbol{\theta}\right)\right] \\
n_{\text {opt }}^{\prime} & =\arg \max _{n} \int b(n, \boldsymbol{\theta}) p(\boldsymbol{\theta}) d \boldsymbol{\theta}
\end{aligned}
$$

Similarly, the maximum posterior expected benefit (PEB) $B^{\prime \prime}(\mathbf{D})$ Rus et al. (2016), which is based on the posterior distribution of the parameters given the data $p(\boldsymbol{\theta} \mid \mathbf{D})$, is obtained as follows Konakli et al. (2015):

$$
\begin{aligned}
B^{\prime \prime}(\mathbf{D}) & =\mathbb{E}_{p(\boldsymbol{\theta} \mid \mathbf{D})}\left[b\left(n_{\text {opt }}^{\prime \prime}, \boldsymbol{\theta}\right)\right] \\
n_{\text {opt }}^{\prime \prime} & =\arg \max _{n} \int b(n, \boldsymbol{\theta}) p(\boldsymbol{\theta} \mid \mathbf{D}) d \boldsymbol{\theta}
\end{aligned}
$$

where the conditioning on $\mathbf{D}$ is to denote that $B^{\prime \prime}$ depends on the data obtained through the sensors. Note that these data can be obtained either from preliminary tests or based on simulations at the design stage, since real data cannot generally be used at this stage. Then, by subtracting both mathematical expectations evaluated at their optimal sensor configurations $n_{o p t}^{\prime \prime}$ and $n_{o p t}^{\prime}$, the conditional value of information (CVI) on $\mathrm{D}$ is given by:

$$
\operatorname{CVI}(\mathbf{D})=B^{\prime \prime}(\mathbf{D})-B^{\prime}
$$

Note that Equation (3) is defined for only one damage scenario, whereby the sensors acquire the data $\mathbf{D}$. In order to obtain a more robust measure that, in turn, provides the optimal sensor configuration given a set of data $\mathfrak{D}$, an expectation of the CVI over the data is required, as follows:

$$
\mathrm{VoI}=\mathbb{E}_{p(\mathbf{D})}[\mathrm{CVI}(\mathbf{D})]
$$

where VoI refers to value of information. The computation of the last expectation requires the solution of the optimal sensor configuration for each of the data $\mathbf{D} \in \mathfrak{D}$ and the expensive calculations of the evidence $p(\mathbf{D})$ Rus et al. (2016) 


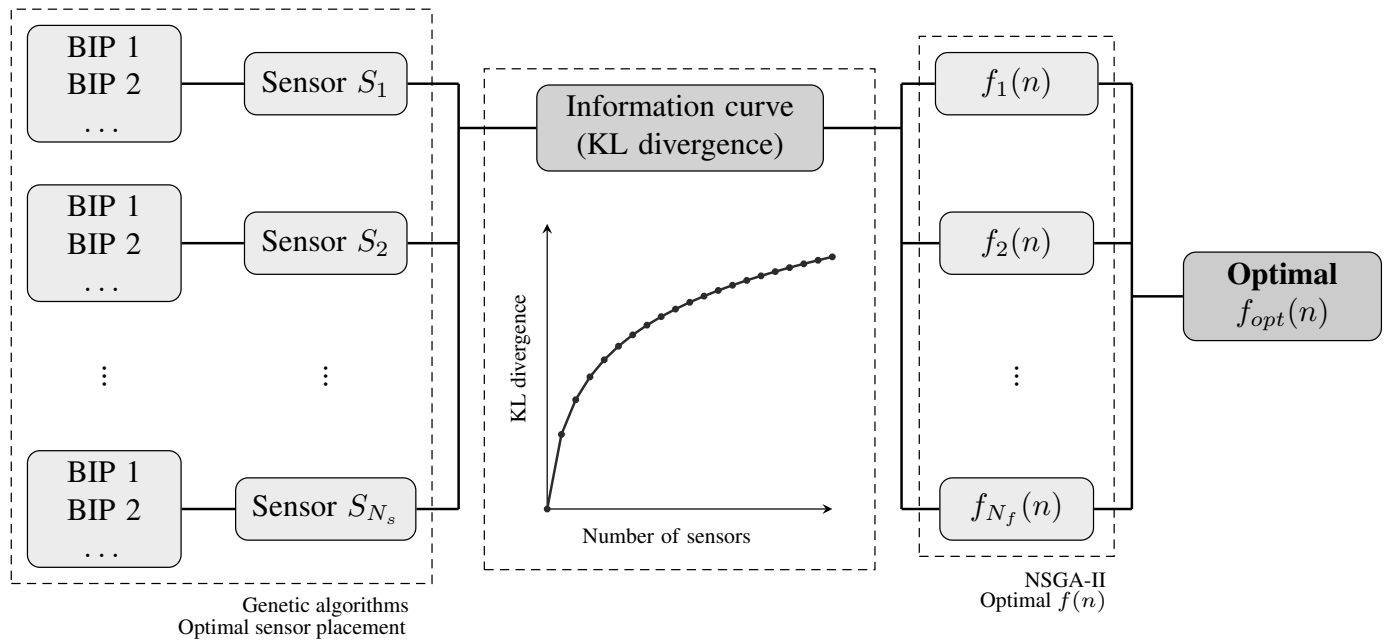

Fig. 1. Optimization approach starting from the construction of the information curve up to the optimization of the inverse cost function $f(n)$. The process goes through two separate optimization algorithms: (1) optimization of the sensor positions by using GA and a discrete problem, and (2) multiobjective optimization of $f(n)$, which should provide the most valuable sensor configuration, while minimizing the costs in terms of number of sensors.

at each sampled data. A pre-posterior analysis, by using data generated by the model and samples coming from the prior distribution of the model parameters would be more appropriate at the design level. However, for the purpose of illustrating the methodology for obtaining the global optimal inverse cost function, the data are restricted here to one simulated damage scenario, leading to the use of CVI for optimal sensor configuration. Finally, note that the sensor placement approach is based on a greedy algorithm that adds sensors one by one as described in Papadimitriou (2004). The sensor location of the newly added sensor is optimized using a genetic algorithm (GA).

\subsection{Optimization of inverse cost function}

The interest behind the optimization of $f(n)$ lies in the potential benefits of obtaining the more convenient inverse cost function that provides a sufficiently informative, yet efficient, optimal sensor configuration. It is worth mentioning that the definition of the inverse cost function $f(n)$ dictates if the optimal number of sensors is on the side of the information, or on the side of the cost of the sensors. For instance, if a $f(n)$ is defined so that it penalizes the addition of sensors in excess, the optimal configuration would be cheap but likely to be insufficiently informative. In contrast, if $f(n)$ does not penalize the use of a relatively large number of sensors, the optimal solution would be highly informative, but possibly impractical in the real world. To autonomously and rigorously select the optimal cost-related function $f_{o p t}(n)$ that provides the best trade-off between information and cost, an approach based on multiobjective genetic algorithms Rothlauf, Franz (2006); Konak et al. (2006) is proposed here.

Figure 1 depicts the proposed methodology to obtain the optimal inverse cost function. First, a curve containing the information gain produced by the optimal sensor layouts using different number of sensors is obtained. Note that these optimal layouts are obtained by using GA, which optimize the sensor locations by maximizing the information gain of the posterior distribution of the damage location. The prior and posterior distributions, which are needed for computing the conditional value of information, are obtained by using model-based Bayesian inverse problems (BIPs) configured in a robust manner, as described in Cantero-Chinchilla et al. (2019). It is worth mentioning that this approach relies on the reconstruction the damage localization. Alternatively, a more detailed information could be obtained by making use of more complex models at a considerably higher computational cost Chiachío et al. (2017). Once the information curve is produced, $f(n)$ can be introduced to obtain a unique optimal sensor configuration. Here, the optimality of $f(n)$ is investigated using three contradictory objective functions, as follows:

a) Maximization of $B^{\prime \prime}(\mathbf{D})$, recall Equation (2). The sought solution should satisfies that $f(n)$ provides the most informative optimal sensor configuration;

b) Minimization of number of sensors $n$. This objective function restricts the potential solution given by a), given that the 
more sensors are used, the more informative the sensor configuration is;

c) Minimization of the dispersion of the PEB curve. This objective function seeks for the uniqueness and identifiability of the optimal solution. To this end, the PEB curve is treated as a probability density function, and the interquartile range, i.e., the range of sensors used between the $25 \%$ and $75 \%$ of the virtual probability, is used to evaluate such dispersion.

Note that the objective function c) is important for supporting rigorous and effective decision making with regards to the SHM sensor configuration. In cases where the PEB curve has several sensor configurations with close PEB values, the selection of one configuration over another may be biased, with important economical and safetyrelated implications. In order for the optimal configuration to be more easily identifiable, the dispersion of the PEB curve is hence minimized by using a specific inverse cost function.

The inverse cost functions investigated in this work are monotonic interpolating functions $f^{(\ell)}(n):\left[0, N_{\max }\right] \rightarrow[0,1]$, which are made of interpolating monotonic cubic splines Fritsch and Carlson (1980) due to its ease of implementation and ability to mimic almost any monotonic function. Note that $N_{\max }$ is the maximum number of sensors. Assuming that the starting and final points $f^{(\ell)}(0)=1$ and $f^{(\ell)}\left(N_{\max }\right)=0$ are fixed, the inverse cost functions are completely defined by introducing an arbitrary number $(\ell)$ of intermediate interpolating points.

\section{Case study}

The methodology is illustrated using a stiffened plate-like structure with a number of predifined sensor locations next to the stiffeners. First, the sensors are optimally placed until $N_{\max }$ is reached, according to a CVI based objective function. Then, a multiobjective optimization is performed to investigate the optimal shape of $f^{\ell}(n)$.

\subsection{Structure description}

The structure used in this case study is a $300 \mathrm{~mm} \times 300 \mathrm{~mm}$ aluminum plate with $50 \mathrm{~mm}$ height stiffeners and $2 \mathrm{~mm}$ thickness for all the elements, as can be observed in Figure 2 The material properties correspond to an aluminum alloy 2024-T351 with Young's modulus $E=73.1 \mathrm{GPa}$, density $\rho=2780 \mathrm{~kg} / \mathrm{m}^{3}$, and Poisson ratio of 0.33 . The guided waves acquired at all the potential sensor locations are simulated using an Abaqus model. To this end, S4R (4-node doubly curved thin or thick shell, reduced integration, hourglass control, finite membrane strains) shell elements ABAQUS (2016) are used along with

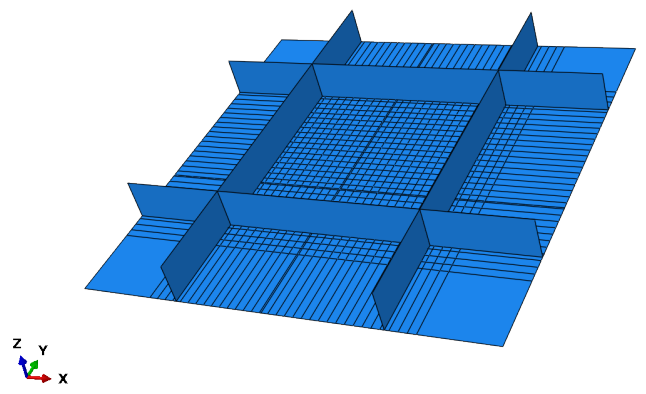

Fig. 2. Abaqus model of the stiffened panel modeled for the purpose of optimal sensor configuration given the simulated data.

a mesh size of $0.5 \mathrm{~mm}$. The guided waves are generated using a 5 cycle sine tone burst centered at a frequency of $300 \mathrm{kHz}$ at the center of the plate in perpendicular direction. Here, the damage is simulated as a $2 \mathrm{~mm} \times 2 \mathrm{~mm}$ hole at $(-19,-49) \mathrm{mm}$, considering the center of coordinate system at the center of the plate. The prior information of the model parameters is assumed to be distributed as follows: $X_{d} \sim \mathcal{U}(-0.1,0) \mathrm{m}$, $Y_{d} \sim \mathcal{U}(-0.1,0) \mathrm{m}$ and $V \sim \mathcal{U}(1500,4500) \mathrm{m} / \mathrm{s}$, where $\mathcal{U}$ refers to a uniform probability density function, $\left(X_{d}, Y_{d}\right)$ are the Cartesian coordinates of the damage, and $V$ is the wave propagation velocity.

\subsection{Multiobjective optimization}

Figure 3 depicts the information curve obtained using the structure described in the previous section and a greedy sensor placement algorithm. As can be observed, the first 3 sensors absorb the majority of information gain. After those sensors, the increase in information rate is drastically reduced. This curve is obtained as the average of computing 100 BIPs with the same sensor configuration and prior, but using different samples. In addition, the $25 \%$ and $75 \%$ percentiles are shown to provide more information about the dispersion. Note also that the higher the number of sensors, the higher the dispersion in the information gain. The mean curve is subsequently used to run the multiobjective optimization algorithm in order to find the optimal inverse cost function.

Using one intermediate interpolating point defined within $n \in[1,30]$ and $f^{(1)}(n) \in[0.01,0.99]$ the optimal inverse cost function obtained is depicted in the upper panel of Figure 4 . Note that the interpolating point is situated at the coordinates $(28.83,0.32)$ and the interpolating cubic spline allows the use of a relatively high number of sensors, i.e. 8 sensors. Such sensor configuration provides the most informative solution at this 


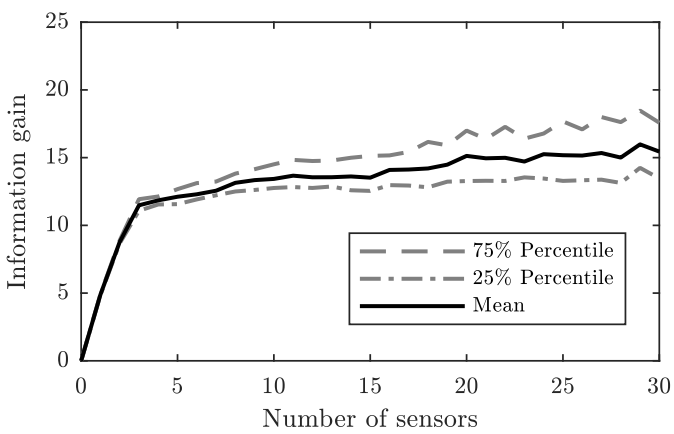

Fig. 3. Mean, $25 \%$, and $75 \%$ percentile of the information curve obtained for all the potential sensor configurations from 0 to $N_{\max }=30$.
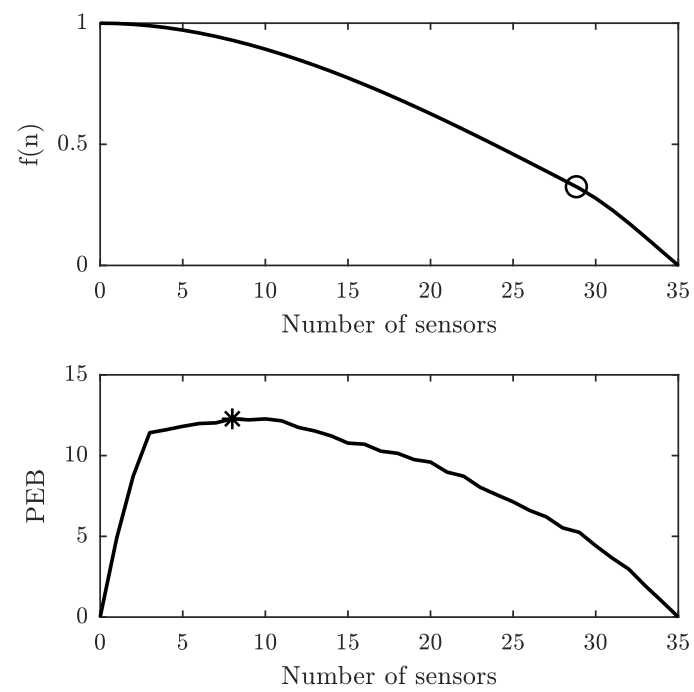

Fig. 4. Optimal inverse cost function using one interpolating point in the upper panel and its corresponding PEB curve in the lower panel.

stage, however, the dispersion of the PEB curve is still relatively high, showing several configurations with a similar PEB value, e.g., between 5 and 10 sensors, as can be observed in the lower panel of Figure 4 This result suggests that the inverse cost function defined by one interpolating point is not enough to comply with the dispersionrelated criterion, thus needing the addition of another interpolating point to modify the inverse cost function $f^{(1)}(n) \rightarrow f^{(2)}(n)$.

The optimal inverse cost function using two intermediate points leads to a more identifiable, yet informative, sensor configuration. The first in-
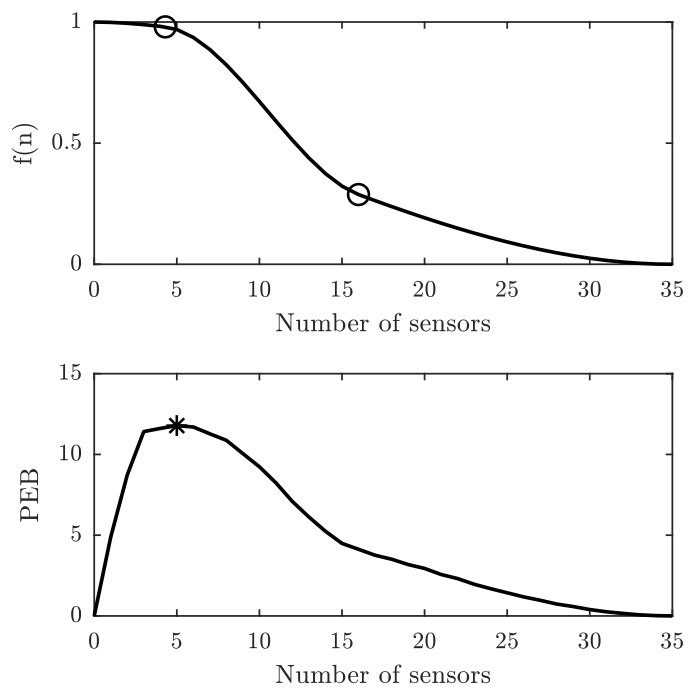

Fig. 5. Optimal inverse cost function using two interpolating point in the upper panel and its corresponding PEB curve in the lower panel.

terpolating point is constrained within $n \in[1,15]$ and $f^{(2)}(n) \in[0.6,0.99]$, while the second point is within $n \in[16,30]$ and $f^{(2)}(n) \in[0.01,0.3]$. The optimal curve is defined by the intermediate points $(4.30,0.98)$ and $(16,0.29)$, as can be observed in the upper panel of Figure 5 . The optimal configuration in this case would be provided by 5 sensors. The use of this type of inverse cost function leads to an optimal sensor configuration with a relatively similar level of PEB, compared to the case with one interpolating point. However, the dispersion is narrower now in the PEB curve shown in the lower panel of Figure 5. This allows the designer to select more clearly the optimal sensor configuration, while minimizing the effects of numerical noise.

\section{Discussion}

The methodology for optimal sensor configuration through the exploration of several inverse cost functions $f(n)$ has been illustrated using monotonic interpolating cubic splines with one and two intermediate anchor points. It has been demonstrated the influence of the definition of $f(n)$ in the selection of the optimal sensor configuration, thus its importance in being optimized. The optimal sensor configuration provided by the optimal $f_{\text {opt }}^{(2)}(n)$ with two interpolating points (upper panel in Figure 5) is generally more useful in the design stage. The selection of the optimal point is less uncertain due to the lower dispersion in the PEB 

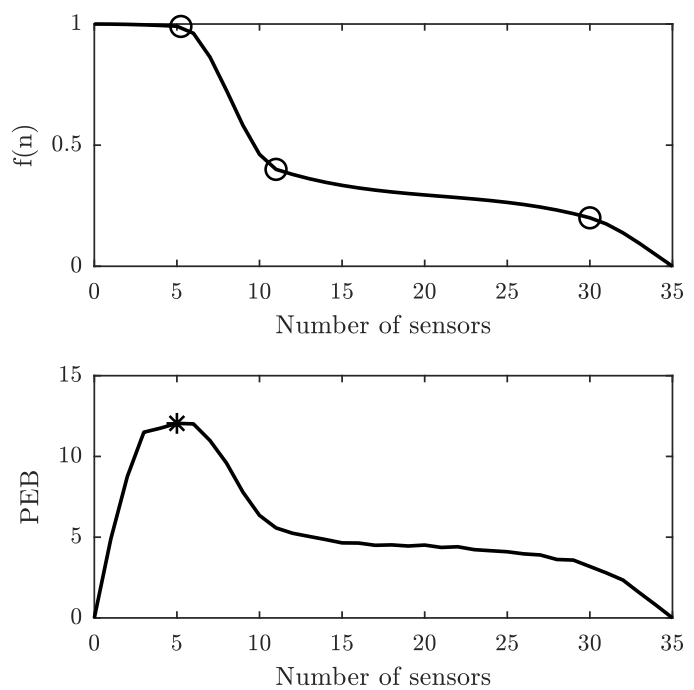

Fig. 6. Optimal inverse cost function using three interpolating point in the upper panel and its corresponding PEB curve in the lower panel.

curve. Furthermore, the $B^{\prime \prime}(\mathbf{D})$ obtained is very similar in absolute value to the one obtained using the $f^{(1)}(n)$ with one interpolating point (upper panel in Figure 4). Therefore, one can assume that the use of the optimal $f_{\text {opt }}^{(1)}(n)$ composed by two interpolating points may be more appropriate for providing balanced optimal sensor configurations between information and cost.

It is also worth mentioning that the definition of the inverse cost function $f^{(3)}(n)$ using three interpolating points does not significantly improve the optimal solution with respect to $f^{(2)}(n)$ composed by two interpolating points. Figure 6 depicts the optimal inverse cost function using three interpolating points and its corresponding PEB curve. As can be observed, both $f_{\text {opt }}^{(3)}(n)$ and the PEB curve are very similar to the ones obtained with two interpolating points. Thus, the usage of the latter would be preferred due to its higher simplicity.

The importance of choosing an optimal inverse cost function before carrying out the design of the SHM system lies in the possibility to devise manufacturing or maintenance processes, so that the informativeness of the SHM system is taken into account before implementing it. Thus, the required equilibrium between the costs and the information that the system is providing can be achieved in an effective and efficient manner.

\section{Conclusions}

A general framework to obtain optimal sensor configurations and inverse cost functions, given the information curve of different sensor configurations, is proposed in this paper. A case study using a stiffened plate-like structure has been used to illustrate the methodology. The adoption of inverse cost functions with three main areas, i.e., composed by two interpolating points, have proven to be effective in providing optimal sensor configurations that comply remarkably well with the three objectives functions: (1) informativeness, (2) cost and (3) identifiability.

The future works include the exploration of the influence of the inverse cost function in the optimal sensor configuration provided over a set of data $\mathfrak{D}$ instead of using one damage scenario $\mathbf{D}$, either by using a pre-posterior analysis or using simulated data from a fast and efficient ultrasonic guided-waves propagation model. In addition, rigorous and efficient optimization procedures for optimal sensor location that rely on theoretic information based approaches will be explored.

\section{Acknowledgements}

This paper is part of the SAFE-FLY project that has received funding from the European Union's Horizon 2020 research and innovation programme under the Marie Sklodowska-Curie grant agreement No 721455. In addition, the authors are grateful for the access to the University of Nottingham High Performance Computing Facility and to the University of Granada for "ROBIN" grant [30.BF.66.11.01], which partially provides support to this work.

\section{References}

ABAQUS (2016). Abaqus Documentation. Providence, RI, USA: Dassault Systèmes.

Argyris, C., S. Chowdhury, V. Zabel, and C. Papadimitriou (2018). Bayesian optimal sensor placement for crack identification in structures using strain measurements. Structural Control and Health Monitoring 25(5), e2137.

Blanloeuil, P., N. A. Nurhazli, and M. Veidt (2016). Particle swarm optimization for optimal sensor placement in ultrasonic SHM systems. In Nondestructive Characterization and Monitoring of Advanced Materials, Aerospace, and Civil Infrastructure 2016, Volume 9804, pp. 9804 - 9804 - 11. International Society for Optics and Photonics.

Cantero-Chinchilla, S., J. Chiachío, M. Chiachío, D. Chronopoulos, and A. Jones (2019). A robust Bayesian methodology for damage localization in plate-like structures using ultrasonic guided-waves. Mechanical Systems and Signal Processing 122, 192-205.

Capellari, G., E. Chatzi, and S. Mariani (2017). 
Optimal sensor placement through Bayesian experimental design: effect of measurement noise and number of sensors. In Multidisciplinary Digital Publishing Institute Proceedings, Volume 1, pp. 41.

Chiachío, J., N. Bochud, M. Chiachío, S. Cantero, and G. Rus (2017). A multilevel Bayesian method for ultrasound-based damage identification in composite laminates. Mechanical Systems and Signal Processing 88, 462-477.

De Stefano, M., M. Gherlone, M. Mattone, M. Di Sciuva, and K. Worden (2015). Optimum sensor placement for impact location using trilateration. Strain 51(2), 89-100.

Deb, K., A. Pratap, S. Agarwal, and T. Meyarivan (2002). A fast and elitist multiobjective genetic algorithm: NSGA-II. IEEE transactions on evolutionary computation 6(2), 182-197.

Fritsch, F. N. and R. E. Carlson (1980). Monotone piecewise cubic interpolation. SIAM Journal on Numerical Analysis 17(2), 238-246.

Howard, R. A. (1966). Information value theory. IEEE Transactions on systems science and cybernetics 2(1), 22-26.

Huan, X. and Y. M. Marzouk (2013). Simulationbased optimal Bayesian experimental design for nonlinear systems. Journal of Computational Physics 232(1), 288-317.

Khodaei, Z. S. and M. Aliabadi (2016). An optimization strategy for best sensor placement for damage detection and localization in complex composite structures. In 8th European Workshop On Structural Health Monitoring (EWSHM 2016), pp. 5-8.

Konak, A., D. W. Coit, and A. E. Smith (2006). Multi-objective optimization using genetic algorithms: A tutorial. Reliability Engineering \& System Safety 91(9), 992-1007.

Konakli, K., B. Sudret, and M. H. Faber (2015). Numerical investigations into the value of information in lifecycle analysis of structural systems. ASCE-ASME Journal of Risk and Uncertainty in Engineering Systems, Part A: Civil Engineering 2(3), B4015007.

Malings, C. and M. Pozzi (2016). Value of information for spatially distributed systems: Application to sensor placement. Reliability Engineering \& System Safety 154, 219-233.

Malings, C. and M. Pozzi (2019). Submodularity issues in value-of-information-based sensor placement. Reliability Engineering \& System Safety 183, 93-103.

Mallardo, V., M. Aliabadi, and Z. S. Khodaei (2013). Optimal sensor positioning for impact localization in smart composite panels. Journal of intelligent material systems and structures 24(5), 559-573.

Papadimitriou, C. (2004). Optimal sensor placement methodology for parametric identification of structural systems. Journal of sound and vibration 278(4-5), 923-947.
Papadimitriou, C., J. L. Beck, and S.-K. Au (2000). Entropy-based optimal sensor location for structural model updating. Journal of Vibration and Control 6(5), 781-800.

Rothlauf, Franz (2006). Representations for Genetic and Evolutionary Algorithms. SpringerVerlag Berlin Heidelberg.

Rus, G., J. Chiachío, and M. Chiachío (2016). Logical inference for inverse problems. Inverse Problems in Science and Engineering 24(3), 448-464.

Salmanpour, M., Z. Sharif Khodaei, and M. Aliabadi (2017). Transducer placement optimisation scheme for a delay and sum damage detection algorithm. Structural Control and Health Monitoring 24(4).

Schlaifer, R. and H. Raiffa (1961). Applied statistical decision theory. MIT Press.

Straub, D., E. Chatzi, E. Bismut, W. Courage, M. Döhler, M. H. Faber, J. Köhler, G. Lombaert, P. Omenzetter, M. Pozzi, et al. (2017). Value of information: A roadmap to quantifying the benefit of structural health monitoring. In ICOSSAR-12th International Conference on Structural Safety \& Reliability.

Tarhini, H., R. Itani, M. A. Fakih, and S. Mustapha (2018). Optimization of piezoelectric wafer placement for structural health-monitoring applications. Journal of Intelligent Material Systems and Structures, 1045389X18799204.

Thiene, M., Z. Sharif-Khodaei, and M. Aliabadi (2016). Optimal sensor placement for damage detection based on ultrasonic guided wave. In Key Engineering Materials, Volume 665, pp. 269-272. Trans Tech Publ.

Thöns, S. (2018). On the Value of Monitoring Information for the Structural Integrity and Risk Management. Computer-Aided Civil and Infrastructure Engineering 33(1), 79-94.

Worden, K. and A. Burrows (2001). Optimal sensor placement for fault detection. Engineering structures 23(8), 885-901. 